\title{
Testing of the ABBN-RF multigroup data library in photon transport calculations
}

\author{
Vladimir Koscheev, Gleb Lomakova , Gennady Manturov, and Anatoly Tsiboulia \\ JSC “SSC RF-IPPE”, Nuclear Reactors and Fuel Cycle Department, 249033 Bondarenko sq. 1, Obninsk, Russia
}

\begin{abstract}
Gamma radiation is produced via both of nuclear fuel and shield materials. Photon interaction is known with appropriate accuracy, but secondary gamma ray production known much less. The purpose of this work is studying secondary gamma ray production data from neutron induced reactions in iron and lead by using MCNP code and modern nuclear data as ROSFOND, ENDF/B-7.1, JEFF-3.2 and JENDL-4.0. Results of calculations show that all of these nuclear data have different photon production data from neutron induced reactions and have poor agreement with evaluated benchmark experiment. The ABBN-RF multigroup crosssection library is based on the ROSFOND data. It presented in two forms of micro cross sections: ABBN and MATXS formats. Comparison of group-wise calculations using both ABBN and MATXS data to point-wise calculations with the ROSFOND library shows a good agreement. The discrepancies between calculation and experimental $\mathrm{C} / \mathrm{E}$ results in neutron spectra are in the limit of experimental errors. For the photon spectrum they are out of experimental errors. Results of calculations using group-wise and point-wise representation of cross sections show a good agreement both for photon and neutron spectra.
\end{abstract}

\section{Introduction}

Accurate knowledge about ionization radiation is very important for nuclear safety study. It can be produced as during operation of nuclear facility in shield materials or transport containers, so in nuclear fuel storage. The main component of this radiation is a photon flux, because the neutron flux is reduced within shield in the most cases. Gamma radiation is produced via both of nuclear fuel and shield materials. Photon interaction data are known with appropriate accuracy, but secondary gamma ray production known much less.

The purpose of this work is to study the photon production data from neutron induced reactions, and testing the data through calculations of ICSBEP Handbook spheres of iron and lead (ALARM-CF-FE-SHIELD-001 and ALARM-CF-PB-SHIELD-001) by using MCNP code and different nuclear data files. Neutron and photon spectra were calculated with the ROSFOND, ENDF/B-7.1, JEFF3.2 and JENDL-4.0 libraries.

The ABBN-RF multigroup cross-section data set, which is based on the ROSFOND nuclear data, in two forms for representation of micro cross sections: standard ABBN and MATXS, was also used in calculations and tested. Comparison of group-wise calculation results obtained by using both ABBN and MATXS data to calculations with ROSFOND point-wise data shows methodical errors group approach.

\section{Benchmarks description}

Two benchmarks from the ICSBEP Handbook: iron and lead spheres of various radii with californium

a e-mail: glomakov@ippe.ru source positioned at the centre of the sphere (ALARMCF-FE-SHIELD-001 and ALARM-CF-PB-SHIELD-001) were used for testing neutron and photon transport and production secondary gamma ray nuclear data.

\subsection{Iron spheres}

In the ALARM-CF-FE-SHIELD-001 benchmark neutron spectra measurements from bare ${ }^{252} \mathrm{Cf}$ source and ${ }^{252} \mathrm{Cf}$ source positioned at the center of 10,15, 20, 25, 30 and $35-\mathrm{cm}$ radius iron spheres were carried out. Photon spectra measurements were done for bare ${ }^{252} \mathrm{Cf}$ source and for ${ }^{252} \mathrm{Cf}$ source positioned at the center of $15,20,25,30$ and $35-\mathrm{cm}$ radius iron spheres.

\subsection{Lead spheres}

In the ALARM-CF-PB-SHIELD-001 benchmark neutron spectra measurements from the ${ }^{252} \mathrm{Cf}$ source positioned at the center of 10, 20 and $30-\mathrm{cm}$ radius lead spheres were carried out. Photon spectra measurements were done for the source positioned at the center of 10,20 and $30-\mathrm{cm}$ radius spheres.

\section{Nuclear data and transport code}

The MCNP5 transport Monte-Carlo code was used in calculations with the recent evaluated nuclear data pointwise libraries.

The ABBN-RF multigroup data set (299groups) retrieved from the ROSFOND nuclear data library was also used.

\subsection{MCNP code}

The MCNP5 is a precise three-dimension Monte Carlo transport code with representation of detail geometry 
of any difficulty. The code is used for solve to tasks of neutron, photon and electron transport. MCNP uses nuclear data presented in the ACE format in both pointwise or group-wise representation.

To compare results of calculations with the benchmark experimental data, the calculation results must be broadened according to the resolution function by Gaussian energy broadening (GEB) in MCNP code.

To compare results of calculations with using pointwise and group-wise libraries it is necessary to turn off photon production by electrons in MCNP (PHYS) because the group data does not have such information. The card PHYS controls energy and physics aspects of MCNP: select IDES $=1$ in cards PHYS:P or PHYS:E (it means that photons will not produce electrons).

\subsection{Nuclear data used}

The ROSFOND2010, JENDL-4.0, ENDF/B-VII and JEFF-3.2 nuclear data libraries were used for calculation neutron transport and production secondary gamma ray in this work. The EPDL-97 data used in all libraries for photon transport.

The ABBN-RF multigroup data set was generated based on the ROSFOND2010 and EPDL-97 nuclear data libraries. The ABBN-RF contains 299 neutron and 127-gamma grouped nuclear data processed by using NJOY [7] code into two formats: ABBN and MATXS [7] formats. The ABBN-RF contains the secondary gamma ray production data only through neutron interactions. The photon production through electron interactions was not included, but this data are necessary for the MCNP code in case of photon calculations.

The CONSYST processing code in case of ABBN data and the TRANSX code for MATXS data were used for calculations of macroscopic cross sections.

\section{Discussion of the results}

The analyses and comparison of the obtained results were performed in two steps:

1. The experimental and calculation data were compared by using point-wise nuclear data libraries for validation of ENDF files;

2. The point-wise and group-wise calculations were compared for determining possible methodical errors in the group approach.

\subsection{Point-wise results}

Comparison of the benchmark neutron source data to the calculation results obtained by using different point-wise nuclear data was considered in the first step of study. The results presented in Fig. 1. We can see that the data are in the limit of experimental uncertainty. For the biggest iron sphere (of radius $35 \mathrm{~cm}$ ) the experimental and calculation results for the neutron leakage spectrum are also in the limits of experimental uncertainty for all nuclear data libraries (Fig. 2).

For the photon leakage spectrum the discrepancy between experimental and calculation results for the biggest iron sphere (Fig. 3) is out of limits of experimental uncertainty in case of all nuclear data libraries. Contribution of primary gammy ray in photon

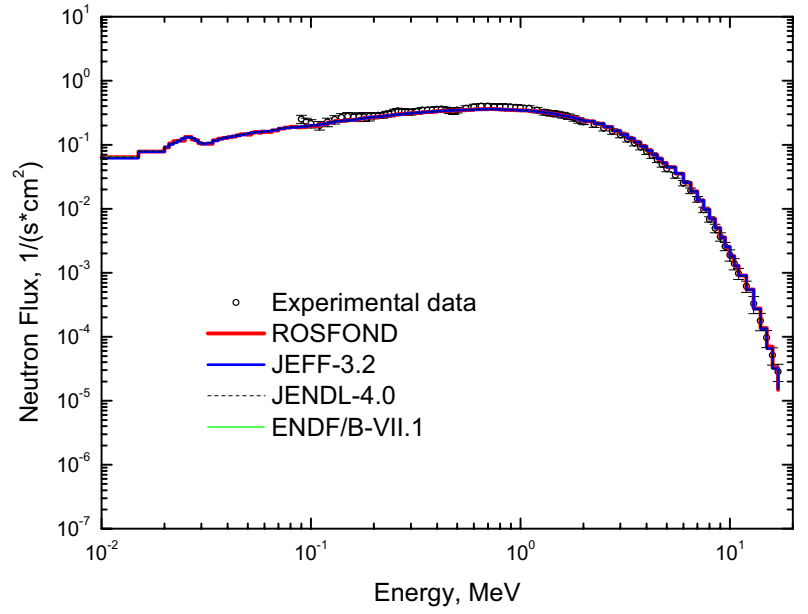

Figure 1. Comparison of experimental and calculation data for neutron leakage spectrum from bare source.

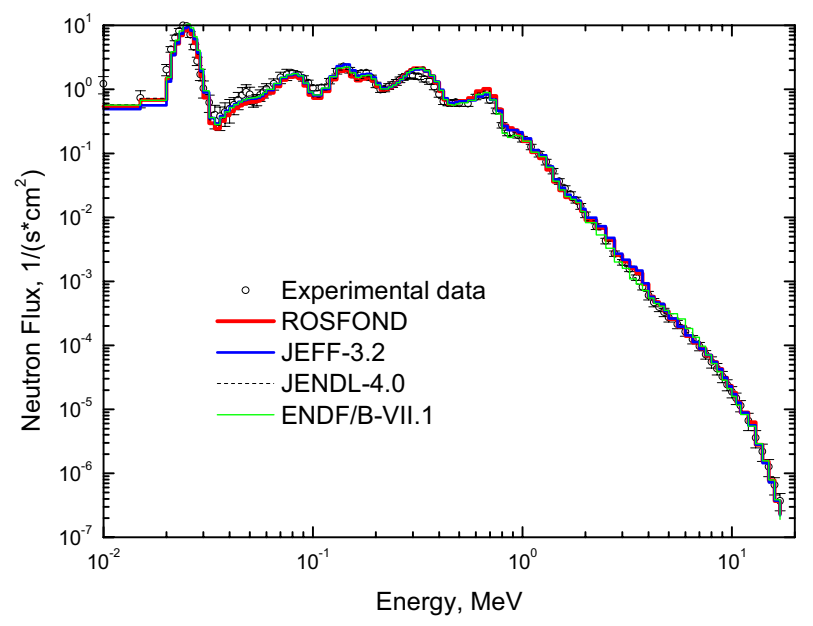

Figure 2. Comparison of experimental and calculation data for neutron leakage spectrum from iron sphere $35 \mathrm{~cm}$ radius.

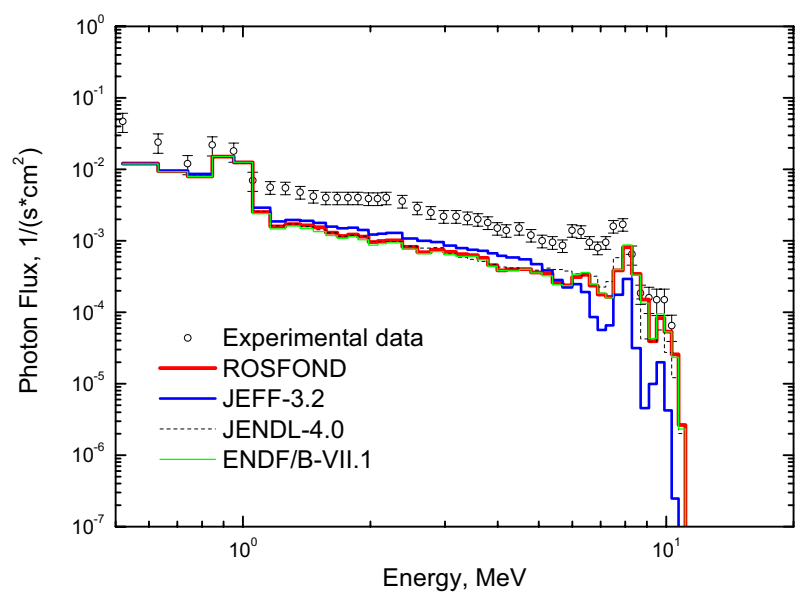

Figure 3. Comparison of experimental and calculation data for photon leakage spectrum from iron sphere $35 \mathrm{~cm}$ radius.

leakage spectrum decreases when radius of spheres increases.

Identical results were obtained for lead spheres (Figs. 4 and 5) for both neutron and photon data. Production secondary gamma ray is insufficient to describe correctly photon leakage spectrum in these benchmarks. 


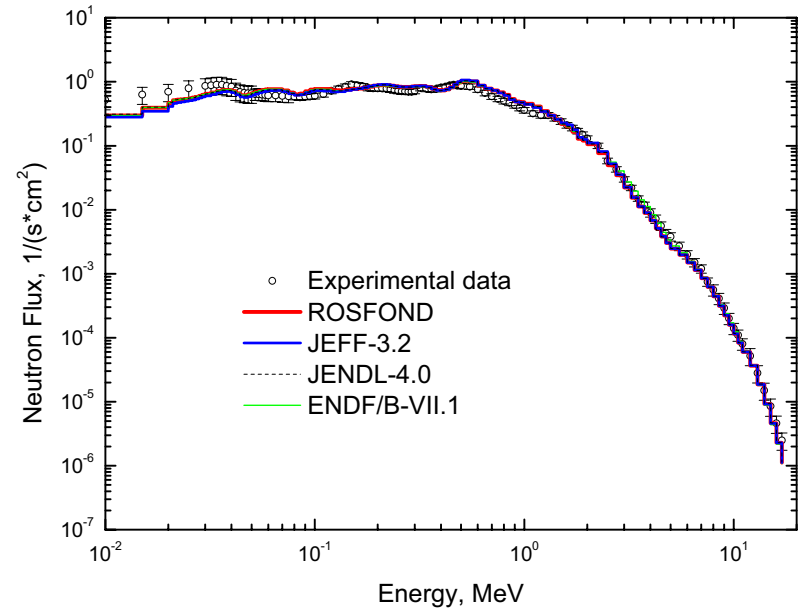

Figure 4. Comparison of experimental and calculation data for neutron leakage spectrum from lead sphere $30 \mathrm{~cm}$ radius.

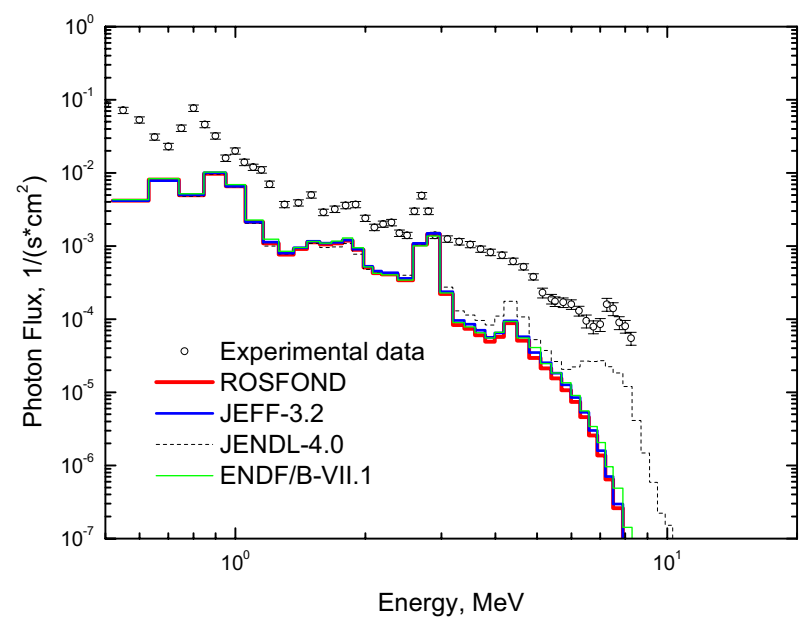

Figure 5. Comparison of experimental and calculation data for photon leakage spectrum from lead sphere $30 \mathrm{~cm}$ radius.

Big discrepancies in photon leakage spectra could be on account of insufficient informational content of experiments or nuclear data. Resolving the this question necessary more of experimental data with production secondary gamma rays. Paper showed first step in this way.

\subsection{Multigroup data}

The obtained calculation results with using ABBN-RF multigroup data were compared to continuous calculations using the ROSFOND nuclear data library.

The Fig. 6 shows the obtained discrepancy in neutron leakage spectrum for spheres of iron (upper) and of lead (lower) between multigroup and continuous calculations.

The group-wise calculations were performed by using two processing systems CONSYST/ABBN-RF and TRANSX/MATXS and as we can see they have small differencies. The Fig. 7 shows the obtained discrepancies in photon leakage spectrum for spheres of iron (upper) and of lead (lower) in multigroup calculations relatively to continuous calculations (fluctuations are related to small particle flux for big spheres).

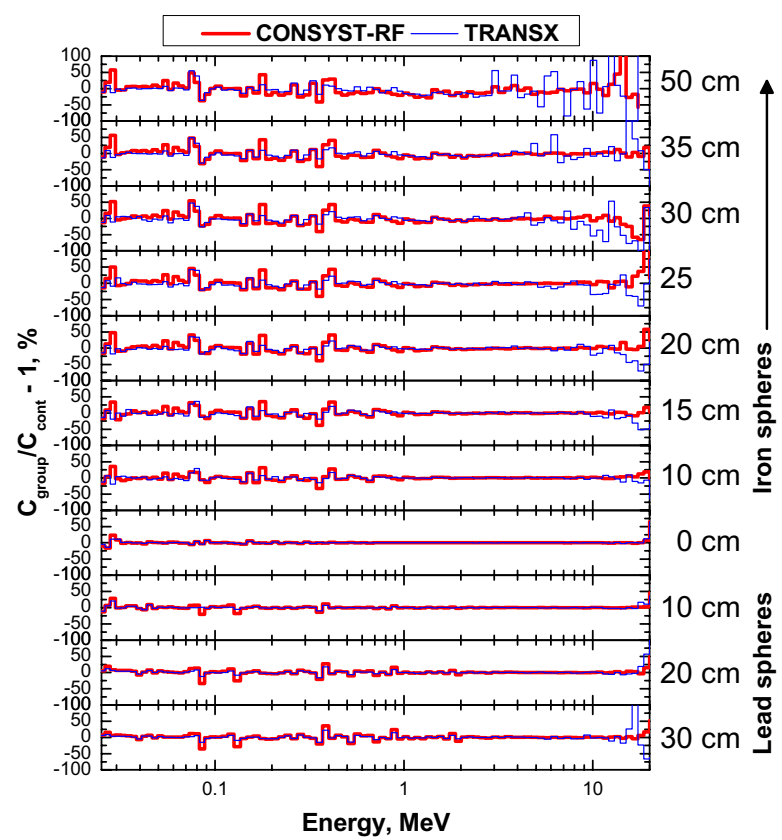

Figure 6. Comparison of calculation data for neutron leakage spectrum for iron (upper) and lead (lower) spheres obtained by using group-wise and point-wise representation of the data.

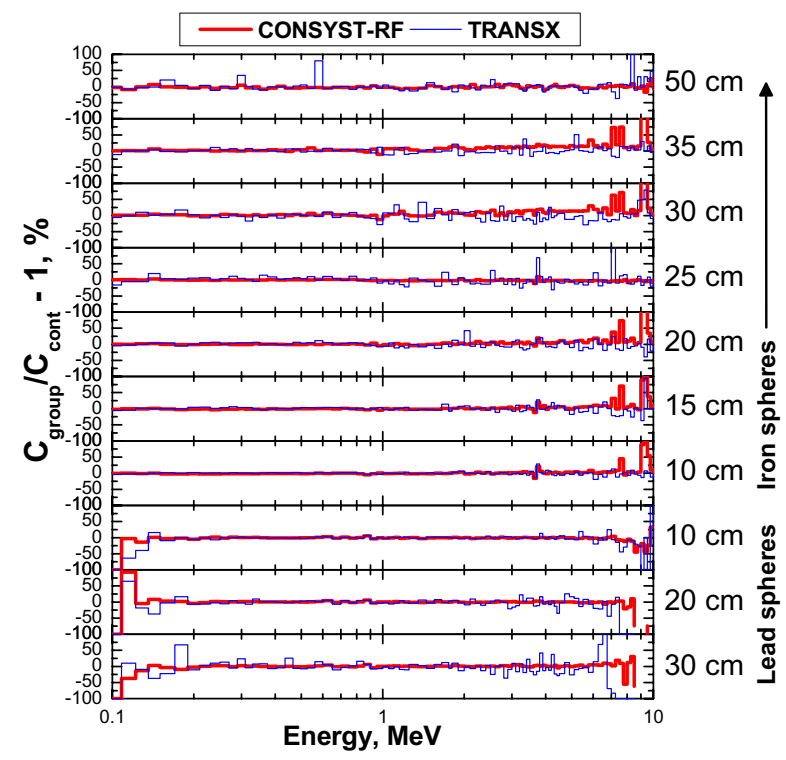

Figure 7. Comparison of calculation data for photon leakage spectrum for iron (upper) and lead (lower) spheres obtained by using group-wise and point-wise representation of the data.

\section{Conclusions}

Comparison of the measurement and calculation results for neutron and photon spectra from bare ${ }^{252} \mathrm{Cf}$ source and the source positioned at the center of iron and lead spheres of different radii, performed by using point-wise ROSFOND and group-wise ABBN-RF nuclear data libraries was done.

Point-wise calculation results showed a good agreement (within one sigma) with experimental data for neutron leakage from surfaces of iron and lead spheres of various radii. Comparison of point-wise calculations with experimental data showed a disagreement (more than three sigma) in photon leakage spectrum from surfaces of iron and lead spheres of various radii. All calculation results 
obtained by using recent nuclear data libraries appeared below experimental data.

When comparing the results of calculations carried out by using point-wise and group-wise data it shows a discrepancy not more than $10 \%$ in neutron and photon spectra, even for the biggest radius of iron and lead spheres.

The authors express their deep appreciation to Trykov L.A. and his team for conducted those significant experiments on the interaction and production of secondary gamma rays in various materials.

\section{References}

[1] International Handbook of Evaluated Criticality Safety Benchmark Experiments, OECD NEA, NEA/NSC/DOC(95)03 (2011)

[2] Trykov L.A., Chernov V.A. PAST. Ser.: NC 1, 71-83 (1997) [in Russian]
[3] Zabrodskaya S.V., Ignatyuk A.V., Koshcheev V.N., Manochin V.N., Nikolaev M.N., Pronyaev V.G. PAST. Ser.: NC 1-2, 3-21 (2007) [in Russian]

[4] MCNP - A General Monte Carlo Neutron-Particle Transport Code. Version 5. X-5 Monte Carlo Team, Los Alamos National Laboratory (April, 2003)

[5] Koscheev V.N., Manturov G.N., Nikolaev M.N., Tsiboulia A.M. IVUZ.YaE 3, 93-101 (2014) [in Russian]

[6] Manturov G.N., Nikolaev M.N., Tsiboulia A.M. PAST. Ser.: NC 1, 59-103 (1996) [in Russian]

[7] R.E. MacFarlane et al., RSIC Peripheral Shielding Routine Collection, PSR-368

[8] Golovko Yu. E., Koscheev V.N., Lomakov G.B., Manturov G.N., Rozhikhin E.V., Semenov M.Y., Tsiboulya A.M., Yakunin A.A. IVUZ.YaE 2, 99-108 (2014) [in Russian]

[9] R.E. MacFarlane, LA-12312-MS (1992) 